18 | 2014

NOVECENTO... E DINTORN

Da Torino a Parigi: Laura Malvano storica e critica d'arte

\title{
Bibliografia di Laura Malvano
}

\section{(2) OpenEdition}

\section{Journals}

Edizione digitale

URL: http://journals.openedition.org/cei/2034

DOI: $10.4000 /$ cei.2034

ISSN: 2260-779X

Editore

UGA Éditions/Université Grenoble Alpes

\section{Edizione cartacea}

Data di pubblicazione: 30 mars 2014

Paginazione: 255-263

ISBN: 978-2-84310-268-4

ISSN: 1770-9571

Notizia bibliografica digitale

"Bibliografia di Laura Malvano», Cahiers d'études italiennes [Online], 18 | 2014, online dal 30 septembre 2015, consultato il 26 mars 2021. URL: http://journals.openedition.org/cei/2034 ; DOI: https://doi.org/ $10.4000 /$ cei.2034 


\section{BIBLIOGRAFIA DI LAURA MALVANO}

\section{6}

Barnaba da Modena, Tesi di laurea, pp. 300, Relatore prof. Anna Maria Brizio, discussa nel corso del mese di novembre.

\section{0}

Stesura di molte voci della Encicolopedia monografica dell'arte, Bologna, Zanichelli.

La mostra di Nicolas de Staël a Torino, "I 4 Soli», anno VII, n 2-3, marzogiugno, pp. 8-15.

L'exposition Dubuffet a Parigi, "Arte Antica e Moderna", n ${ }^{\circ}$ I2, ottobredicembre, pp. 455-457.

1962

Zoltan Kemeny, «Arte Antica e Moderna», n ${ }^{\text {17}}$, gennaio-marzo, pp. 23-24. Bonnard e Vuillard, "Arte Antica e Moderna», n I7, gennaio-marzo, p. 28. I "Picasso di Vauvenargues», "Arte Antica e Moderna», $\mathrm{n}^{\circ}$ I7, gennaiomarzo, p. 29.

XXXI Biennale Internazionale d'Arte di Venezia. I padiglioni stranieri, "Arte Antica e Moderna», n I8, aprile-giugno, pp. 22-23.

Sculture di Max Ernst, "Arte antica e moderna», n 19 , luglio-settembre, pp. 25-28.

Mortensen, "Arte antica e moderna», $\mathrm{n}^{\circ}$ 19, luglio-settembre, p. 29.

Vasarely, "Arte antica e moderna», ${ }^{\circ}$ I9, luglio-settembre, p. 30.

Moore, «Arte antica e moderna", $\mathrm{n}^{\circ}$ 19, luglio-settembre, p. $3 \mathrm{I}$.

Pignon, "Arte antica e moderna», $\mathrm{n}^{\circ}$ I9, luglio-settembre, p. 32.

Alechinsky, "Arte antica e moderna», $\mathrm{n}^{\circ}$ 20, ottobre-dicembre, pp. I6-2I.

Rothko, "Arte antica e moderna", $\mathrm{n}^{\circ}$ 20, ottobre-dicembre, p. 25.

Kokoschka, "Arte antica e moderna», $\mathrm{n}^{\circ}$ 20, ottobre-dicembre, p. 28. 
Dopo 2000 anni le sfingi fenicie vengono ad affascinare i parigini, "Avanti!», 9 agosto.

Schwitters, "Arte antica e moderna», $\mathrm{n}^{\circ} 22$, aprile-giugno, p. 8 .

De Staël, "Arte antica e moderna», $n^{\circ} 23$, luglio-settembre, pp. 4-5.

Delacroix, «Arte antica e moderna», $\mathrm{n}^{\circ} 23$, luglio-settembre, p. 8 .

Kandinsky, "Arte antica e moderna», $\mathrm{n}^{\circ}$ 23, luglio-settembre, pp. II-I2.

Mostra di "l'Art dans l'Occident romain" al Louvre, "Arte antica e moderna», $\mathrm{n}^{\mathrm{o}} 23$, luglio-settembre, pp. 258-259.

Mostra di Charles Le Brun al castello di Versailles, "Arte antica e moderna», $\mathrm{n}^{\mathrm{o}} 23$, luglio-settembre, pp. 259-261.

Parigi - III Biennale, "Arte antica e moderna», $\mathrm{n}^{\circ} 24$, ottobre-dicembre, p. I4.

$N$. Gontcharova et M. Larinov, "Arte antica e moderna», $\mathrm{n}^{\circ} 24$, ottobredicembre, p. I6.

L'Au-delà dans l'Art japonais, "Arte antica e moderna", n²4, ottobredicembre, p. 17.

$$
1964
$$

L'Art au Pays des Hittites, "Arte antica e moderna", n 25, gennaio-marzo, p. 3.

Goya, «Arte antica e moderna», $\mathrm{n}^{\circ}$ 25, gennaio-marzo, pp. 4-6.

Ruskin and his circle, "Arte antica e moderna", $\mathrm{n}^{\circ} 25$, gennaio-marzo, p. 7 . Donation Delaunay, "Arte antica e moderna», $\mathrm{n}^{\circ} 25$, gennaio-marzo, p. 13. Signac, "Arte antica e moderna», $\mathrm{n}^{\circ}$ 25, gennaio-marzo, pp. I3-I4.

LONDRA: Gallerie, "Arte antica e moderna», $\mathrm{n}^{\circ} 25$, gennaio-marzo, pp. I4-I5.

L'esposizione di Picasso a Parigi, "Il Contemporaneo», n 69, febbraio, pp. I09-III.

Preziosi quadri di Sonia e Robert Delaunay in mostra al Louvre, "Avanti!», 4 marzo.

L'interessante mostra delle "Mythologies quotidiennes», "l'Unità», I5 agosto, p. 8.

Toulouse-Lautrec al Petit Palais, "Paese Sera», I9 dicembre.

Lettera da Parigi. Géricault pittore moderno senza ufficialità, "l'Unità», 20 dicembre, p. 8. 


\section{5}

La tecnica del "collage» in una mostra a Parigi, "Paese Sera», 7 gennaio. L' "École de Paris» si difende, «l'Unità», 9 gennaio, p. 6.

Lettera da Parigi. É esplosa la stagione delle grandi mostre ufficiali, "l'Unità», 27 febbraio, p. 6.

Lettera da Parigi. Tesori delle chiese di Francia, "l'Unità», I3 marzo, p. 6. Lettera da Parigi. L'ordine luminoso di Nicolas de Staël, «l'Unità», 3 aprile, p. 6.

I capolavori della pittura francese nei musei sovietici, "l'Unità», I9 giugno. Una grande trilogia di Matta: "Lo stato dell'Unione», "l'Unità», 26 giugno. Lettera da Londra. L'uomo che cammina di Giacometti. L'uomo "atomico» di Henri Moore.

L'uomo crocifisso di Bacon, "l'Unità», 2I settembre, p. 6.

Pissarro, Milano, Fratelli Fabbri Editore.

\section{6}

Courbet, Milano, Fratelli Fabbri Editore.

Lettera da Parigi. Un'antologia "ufficiale» di pittori italiani, "l'Unità», 26 giugno, p. I2.

Grande retrospettiva di Gustave Courbet, «l'Unità», I3 luglio, p. 8.

Cento opere di giovani artisti toscani, «l'Unità», 28 settembre, p. 9.

Al Museo delle Arti Decorative di Parigi. L'arte moderna alla Peter Stuyvesant, «l'Unità», 20 dicembre, p. 8.

\section{7}

Lettera de Parigi. Visita alla V Biennale Internazionale. L'arte in serie in vendita nei grandi magazzini, "l'Unità», 27 dicembre, p. 8.

Per la prima volta a Parigi una retrospettiva di Bonnard, "Paese Sera", 22 marzo.

Riuniti insieme a Parigi i "pittori di contestazione», "Paese Sera», 27 luglio. Una "parata mondana» nei quadri di Van Dongen, "Paese Sera», 5 dicembre.

1968

Quando l'arte si può gonfiare, "l'Unità», 8 maggio, p. 8.

Lettera da Parigi. Gli artisti francesi dopo il "maggio", "l'Unità», 25 ottobre, p. 3 . 
Timide aperture nel "protezionismo" artistico francese. L'arte negli Stati Uniti tra il 1948 e il 1968. La "Morte del padre», nuova grande scultura di Jean Ipoustéguy esposta da Claude Bernard, «l'Unità», 28 dicembre, p. 9.

\section{9}

Une vierge à l'enfant de Barnaba da Modena, "Revue du Louvre», n 6, pp. 339-346.

Le donne-mostri di Brauner, "l'Unità», I3 febbraio, p. I2.

Lettera da Parigi. Omaggio a Baudelaire, «'Unità», Is febbraio, p. Io.

Giacometti all'Orangerie, «'Unità», 2I novembre, p. Io.

Lettera da Parigi. Il cosmo dell'immaginazione... una grande mostra di Paul Klee..., «l'Unità», 24 dicembre, p. I2.

\section{0}

Da Ensor a Permeke: il contributo fiammingo alla pittura moderna, «'Unità», 3 maggio, p. I4.

Una grande mostra celebrativa di Henri Matisse a Parigi. Il pittore della gioia di vivere, «'Unità», IO maggio, p. I4.

\section{1}

Naturalismo e realismo, in Giovanni Previtali (a cura di), Arte 2 (Enciclopedia Feltrinelli Fischer), Milano, Feltrinelli, pp. 353-442.

\section{2}

Pierre Bonnard, la vie et l'cuvre [Il libro, già consegnato alle Éditions Rencontre di Losanna, non è mai stato pubblicato perché l'editore ha cessato le sue attività].

\section{6}

(con Antonio Bechelloni): Louis-Philippe: l'homme et le roi, in «Prospettiva», $\mathrm{n}^{\circ} 4$, giugno, pp. 62-65.

Les paysages de P. H. de Valenciennes, in «Prospettiva», n 6, luglio, pp. 64-69.

\section{7}

Le Symbolisme en Europe, in "Prospettiva ", no 8, gennaio, pp. 72-77.

Futurisme et fascisme : dynamique de rapports inégaux, in Giovanni Lista (a cura di), Marinetti et le futurisme, Lausanne, L’Âge d'Homme, pp. I62-I7I. 
Saint-Simon et la partie poétique du nouveau système, in Scritti per Maria Cionini-Visani, Torino, pp. I57-I60.

\section{8}

Le débat autour du Réalisme entre I855 et 1865, "Histoire e Critique des Arts", no 4-5, maggio, pp. 62-69.

Note critique à partir du texte de F. Antal, "Histoire et Critique des Arts», $\mathrm{n}^{\circ}$ 7-8, dicembre, pp. 19-20.

Le débat sur les Musées en Italie, ivi, pp. I06-I07.

$$
1979
$$

Neo-Dada, in Appendice IV, Enciclopedia Treccani.

Petit Larousse de la Peinture, Paris: 93 voci su artisti, movimenti, strutture artistiche dell'arte italiana del XX secolo.

\section{0}

Le sujet politique en peinture : événements et histoire pendant les années de la Révolution, "Histoire et Critique des Arts», n I3-I4, pp. 3I-66.

$$
\text { 198I }
$$

Pop-art, in Appendice IV, Enciclopedia Treccani. Andy Warhol, in Appendice IV, Enciclopedia Treccani. Realismo-iperrealismo, in Appendice IV, Enciclopedia Treccani.

\section{4}

P. Bourdieu, "La distinzione, critica sociale del giudizio». Il Mulino, Bologna, in «Prospettiva», $\mathrm{n}^{\circ} 38$, luglio, pp. 86-90.

\section{6}

Fascismo e politica dell'immagine. Note per una ricerca, "Mezzosecolo», $\mathrm{n}^{\circ}$ 6, annali 1985-1986, pp. 219-225.

$$
1987
$$

Structures sociales et production figurative : l'art italien entre avant-garde et dirigisme culturel, tesi di dottorato, discussa all'Università di Paris 8. Composizione della giuria: Françoise Decroisette, Pierre Milza, Fanette Roche, Giuditta Rosowsky. 
1988

Fascismo e politica dell'immagine, Torino, Bollati-Boringhieri.

La Peinture murale, lieu privilégié de la politique fasciste de l'image, in «Ligeia, dossiers sur l'art», $n^{\circ}$ I, aprile-giugno, pp. 56-64.

\section{9}

La politique artistique dans un régime totalitaire. Le cas du fascisme, in Pierre Milza e Fanette Roche-Pézard (a cura di), Art et Fascisme. Totalitarisme et résistance au totalitarisme dans les arts en Italie, Allemagne et France des années 30 à la défaite de l'Axe, Bruxelles, Éditions Complexe, pp. I55-I79.

\section{0}

Note sulla fortuna critica del "Marat» di David, in Scritti in ricordo di Giovanni Previtali, vol. II, «Prospettiva», n 57-60, aprile 1989-ottobre I990, pp. 369-376.

Francis Bacon, in Encyclopaedia Universalis, Paris.

I991

La mostra della Rivoluzione, in Françoise Liffran (a cura di), Rome I9201945, Le modèle fasciste, son Duce, sa mythologie, "Autrement", $\mathrm{n}^{\circ} 7$, aprile, pp. I45-I53.

\section{4}

Il mito della giovinezza attraverso l'immagine: il fascismo italiano, in Giovanni Levi e Jean-Claude Schmitt (a cura di), Storia dei giovani. 2. L'età contemporanea, Roma-Bari, Laterza, pp. 3II-348.

L'événement politique en peinture. À propos du Marat de David, "Mélanges de l'École française de Rome. Italie et Méditerranée», t. Io6, nº I, pp. 33-54.

\section{5}

Entre les contraintes d'un art officiel et les attraits du marché : le cas Melotti, in Mady Ménier (a cura di), De la métaphysique au physique, Hommage à Fanette Roche-Pézard, Publications de la Sorbonne, pp. 9I-IO5.

Entre la Révolution française et le fascisme italien: considérations autour de l'"art social», "Bulletin de l'Association des histoires de l'art italien», $\mathrm{n}^{\mathrm{o}}$ I, pp. 7-8. 
1996

Le mythe de la jeunesse à travers l'image. Le fascisme italien, in Giovanni Levi e Jean-Claude Schmitt (a cura di), Histoire des jeunes en Occident, t. 2, L'époque contemporaine, Paris, Seuil, pp. 277-308 (traduzione dall'italiano dell'articolo Il mito della giovinezza, cit.).

Le Style en question, in «Ligeia, dossiers sur l'art», $\mathrm{n}^{\circ}$ I7-I8, ottobre $1995-$ giugno 1996, pp. I5-I7.

1997

The Myth of Youth in Images: Italian Fascism, in Giovanni Levi e JeanClaude Schmitt, (a cura di), A History of Young People in the West, vol. 2: Stormy Evolution to Modern Times, trans. Carol Volk, London and Cambridge, Mass., Belknap Press of Harvard University, pp. 232-256 (trad. dall'italiano de Il mito della giovinezza, cit.)

\section{8}

I ritratti del duce sono migliaia: note per una storia dei ritratti del duce, in «Dialoghi di Storia dell'Arte», nº 7, pp. I37-I40.

1999

Piero Gobetti critico d'arte: "dignità» e "buon costume» alle prese con l'«arte reggitrice di Stato", in Michel Cassac (a cura di), Piero Gobetti et la culture des années 20, Nizza, Publications de la Faculté des Lettres, Arts et Sciences Humaines, pp. 215-238.

2000

Piero Gobetti critico d'arte: la «dignità dell'arte» come arma d'opposizione nella Torino degli anni venti, in "Prospettiva», $\mathrm{n}^{\circ}$ 98-99, aprile-luglio, pp. 209-220.

L'utopie de l'art social au XIX siècle: Saint-Simon et «la partie poétique du nouveau système», in Roberto Barbanti e Claire Fagnart (a cura di), L'Art au XX siècle et l'utopie : réflexions et expériences, Paris, l'Harmattan, pp. 215-224.

Il tema del lavoro negli anni del Realismo, in F. Poli, G. Anzani e L. Malvano (a cura di), Catalogo della mostra "Arte e Lavoro, Dal Verismo al Neorealismo», Aosta, Elede. 
La représentation de la "modernité" dans l'art du XX siècle : l'image de la ville, «Ligeia, dossiers sur l'art», $\mathrm{n}^{\circ}$ 33-36, ottobre-giugno, pp. 23-36.

Il pittore e il critico: note sulla ricezione della prima monografia casoratiana, in Rosanna Maggio-Serra (a cura di), Piero Gobetti e Felice Casorati, I9I8-1926, Catalogo della mostra tenuta a Torino, 30 ottobre-2 dicembre 200I, Ministero per i Beni e le Attività Culturali, Direzione Generale degli Archivi di Stato, Milano, Electa, pp. 23-30.

2002

L'«art social» en Italie : l'image du Peuple entre événement et Histoire, «Bulletin de l'Association des histoires de l'art italien», nº 7, pp. 6I-67.

2003

Prendi l'arte e mettila da parte, "Il Manifesto», 25 marzo, p. I5.

De "la fiumana dell'Umanità assetata di giustizia" à la foule consensuelle du fascisme: à propos de la représentation de la foule en peinture, in "Laboratoire italien", $\mathrm{n}^{\circ} 4$, pp. 95 -IIO.

"Les petits sujets» e la grande decorazione murale: Edouard Vuillard tra "reconstitution du naturel» $e$ "arts de surface», in "Prospettiva», $\mathrm{n}^{\circ}$ IIO-III, aprile-luglio, pp. I69-178.

Le mythe de la romanité et la politique de l'image dans l'Italie fasciste, in "Vingtième siècle», giugno, pp. III-I2O.

Les Baigneuses de Courbet: La "grâce féminine" vue par un "fort de la Halle en peinture», in "Ligeia, dossiers sur l'art», $\mathrm{n}^{\circ} 4 \mathrm{I}-44$, ottobre 2002giugno 2003, pp. 216-223.

Le futurisme: l'art d'avant-garde en Italie entre les deux guerres, in De Chirico et la peinture italienne de l'entre-deux-guerres. Du Futurisme au retour à l'ordre, catalogo della mostra del Musée de Lodève, Hôtel du Cardinal de Fleury, 4 luglio-26 ottobre 2003, Cinisello Balsamo (Milano), Silvana Editoriale, pp. 49-75.

2006

Utopie d'un projet et contraintes d'une pratique : l'art social en France entre deux révolutions, in A. Cohen, $\mathrm{R}$. Congost e P. F. Luna (a cura di), Pierre Vilar : une histoire totale, une histoire en construction, Paris, Éd. Syllepse, pp. I55-I68. 
«Salvare i giorni della vita dalla dimenticanza». Pittura e scrittura nell'opera di Nella Marchesini Malvano, in A. Malvano, L. Malvano e P. Mantovani (a cura di), Nella Marchesini, catalogo della mostra della Galleria del Ponte a Torino, 9 febbraio-3I marzo 2006, Torino, Moglia s.r.l. Tipolitografia, s.d. (ma 2006).

\section{7}

Realismi, in F. Poli (a cura di), Arte moderna. Dal post-impressionismo all'informale, Milano, Electa, pp. 242-26r. 